\title{
Synthesis of benzothiophene and indole derivatives through metal-free propargyl-allene rearrangement and allyl migration
}

\author{
Jinzhong Yao*, Yajie Xie, Lianpeng Zhang, Yujin Li and Hongwei Zhou*
}

\author{
Full Research Paper \\ Address: \\ College of Biological, Chemical Sciences and Engineering, Jiaxing \\ University, Jiaxing 314001, P. R. China \\ Email: \\ Jinzhong Yao* - jzyao@zju.edu.cn; Hongwei Zhou* - \\ zhouhw@zju.edu.cn \\ * Corresponding author \\ Keywords: \\ allyl migration; benzothiophene; indole; metal-free; propargyl-allenyl \\ Beilstein J. Org. Chem. 2017, 13, 1866-1870. \\ doi:10.3762/bjoc. 13.181 \\ Received: 12 May 2017 \\ Accepted: 07 July 2017 \\ Published: 06 September 2017 \\ Associate Editor: D. Spring \\ (C) 2017 Yao et al.; licensee Beilstein-Institut. \\ License and terms: see end of document.
}

\begin{abstract}
An efficient base-catalyzed protocol for the synthesis of benzothiophene is described. The reaction proceeds via base-promoted propargyl-allenyl rearrangement followed by cyclization and allyl migration. Phosphine-substituted indoles can be synthesized by a similar strategy.
\end{abstract}

\section{Introduction}

Heterocycles are frequently found in natural products and pharmacologically active compounds, thus economic and efficient methods to construct heterocycles are always highly desirable [1-6].

Benzothiophenes are important heterocycles that are one of the key motifs of anti-inflammatory, anti-estrogenic and anti-HIV drugs (Figure 1) [7-9]. Moreover, benzothiophenes have extensive applications in materials science. Besides the traditional methods of transition metal-catalyzed cyclization of alkyne substrates [10-12], the synthesis of benzothiophenes via metal-free conditions has recently aroused much attention [13-15]. For example, the preparation of C3-borylated benzothiophene by $\mathrm{BCl}_{3}$-induced borylative cyclization of arylalkynes was recently demonstrated by Ingleson [16].
Allene-mediated cyclization reactions are advantageous due to the convenient preparation of starting materials instead of the use of unstable or reactive polyfunctionalized allene substrates [17-27]. Although transition metal (e.g., Au, Pd)-catalysed propargyl-allenyl isomerization and cyclization reactions have been established $[28,29]$, such transformations promoted by a base to construct heterocycles are not well-documented [30,31]. Recently, our group explored the utilization of $\beta$-sulfonium carbanions for the preparation of thiophene derivatives [19]. Alkynes were treated with acyl chloride under Sonogashira reaction conditions and the expected $\beta$-sulfonium carbanions were obtained in a one-pot process. Based on our understanding of organosulfur chemistry [20-22], we report herein a simple, metal-free method for the formation of benzothiophenes using an intramolecular addition of a sulfur atom (origi- 
<smiles>CC(c1cc2ccccc2s1)N(O)C(N)=O</smiles>

zileuton anti-inflammatory

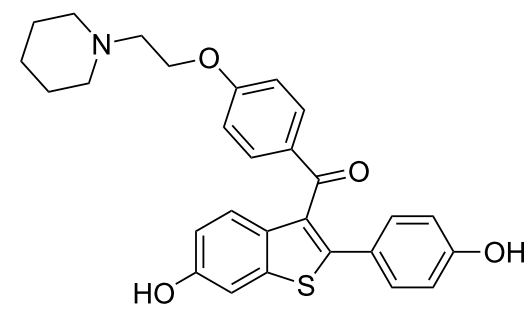

raloxifene anti-estrogenic<smiles>COC(=O)C1=C(N2CCCCC2)C2c3ccccc3SC12C(=O)OC</smiles>

NSC-380292 anti-HIV

Figure 1: Examples of biologically active benzothiophene derivatives.

nated from a sulfide) to the electron-deficient allene moiety generated in situ by a propargyl-allenyl rearrangement [17-27] and an allyl migration [32-34] (Scheme 1). In addition, phosphine-substituted indole derivatives could also be conveniently constructed by a similar strategy. This method not only avoids the use of transition metal catalysts, but also provides the useful heterocycles which are not easily achieved through other protocols.<smiles>[R]C#CC([R])c1ccccc1SCC=C</smiles>

1<smiles>[R]c1c(C([R])CC=C)sc2ccccc12</smiles>

2<smiles>[R]C=C1C[SH](CC=C)c2ccccc2C1[R]</smiles>

intramolecular nucleophilic attack

\section{[3,3] sigmatropic} rearrangement

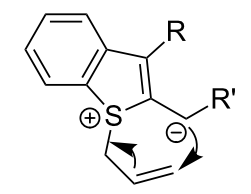

Scheme 1: Proposal of applicable $\beta$-sulfonium carbanion.

\section{Results and Discussion}

In the initial studies, we treated methyl 4-(3-(2-(allylthio)phenyl)-3-methoxyprop-1-yn-1-yl)benzoate (1a) with DBU ( 0.1 equiv) in THF at $50{ }^{\circ} \mathrm{C}$ under $\mathrm{N}_{2}$ for $12 \mathrm{~h}$ (Table 1 , entry 1). Fortunately, the desired product $\mathbf{2 a}$ was obtained in $57 \%$ yield. No reaction was observed using TEA or DABCO, possibly because the allenic intermediate could not be formed by these comparatively weak bases (Table 1, entries 2 and 3), which was different from the previous work. Other bases, such as $\mathrm{TBD}, \mathrm{Cs}_{2} \mathrm{CO}_{3}$, and $t$-BuOK were found to be less effective (Table 1, entries 4-6). To our delight, it was found that increasing the catalyst loading to 0.2 equiv resulted in an obviously higher yield of $83 \%$ (Table 1, entry 7). However, a higher catalyst loading had almost no influence on the reaction (Table 1 , entry 8). It was found that THF was the best solvent after screening different solvents. Other solvents, such as DCE, toluene, and $\mathrm{CH}_{3} \mathrm{CN}$ were found to be less effective (Table 1, entries 9-11). The yield was reduced to $51 \%$ when the reaction time was decreased to $6 \mathrm{~h}$ (Table 1, entry 12). A lower temperature was found to be less effective for the reaction (Table 1, entry 13). Without the base, no reaction occurred, implying that the reaction proceeded exclusively through the allenic intermediate (Table 1, entry 14). Thus, the optimal reaction conditions were DBU ( 0.2 equiv) under nitrogen in THF at $50{ }^{\circ} \mathrm{C}$ for $12 \mathrm{~h}$.

Table 1: Optimization of the reaction conditions ${ }^{a}$.

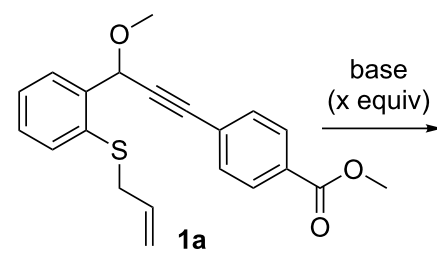<smiles>C=CCC(c1ccc(C(=O)OC)cc1)c1sc2ccccc2c1OC</smiles>

\begin{tabular}{lllll}
\hline Entry & Catalyst & $\mathrm{x}$ & Solvent & yield $(\%)^{\mathrm{b}}$ \\
\hline 1 & DBU & 0.1 & THF & 57 \\
2 & TEA & 0.1 & THF & N.D \\
3 & DABCO & 0.1 & THF & N.D \\
4 & TBD & 0.1 & THF & 22 \\
5 & $\mathrm{Cs}_{2} \mathrm{CO}_{3}$ & 0.1 & THF & 23 \\
6 & $t$-BuOK & 0.1 & THF & 27 \\
7 & DBU & 0.2 & THF & 83 \\
8 & DBU & 0.5 & THF & 82 \\
9 & DBU & 0.2 & DCE & 62 \\
10 & DBU & 0.2 & toluene & 68 \\
11 & DBU & 0.2 & CH ${ }_{3} \mathrm{CN}$ & 58 \\
12 & DBU & 0.2 & THF & $51^{\mathrm{c}}$ \\
13 & DBU & 0.2 & THF & $32^{\mathrm{d}}$ \\
14 & - & - & THF & $\mathrm{N} . \mathrm{D}$ \\
\hline
\end{tabular}

aReaction conditions: 1a (1.0 equiv), base (x equiv), $50{ }^{\circ} \mathrm{C}, 12 \mathrm{~h}$, under

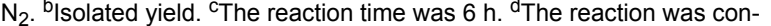
ducted at $25^{\circ} \mathrm{C}$. DBU: 1,8-diazabicyclo[5.4.0]undec-7-ene. TBD: 1,5,7triazabicyclo[4.4.0]dec-5-ene. 
With the optimized reaction conditions in hand, we turned our attention to study the reaction scope and limitations of this reaction; the results are shown in Figure 2. A series of alkynes substituted with an electron-withdrawing group participated in this reaction smoothly to give the products in good yields $(\mathbf{2 a}-\mathbf{k})$. A variety of substituents, such as $p$-COOEt, $p-\mathrm{COCH}_{3}$, dichloro, $p-\mathrm{NO}_{2}, p-\mathrm{CF}_{3}$ and $p-\mathrm{CN}$ were well-tolerated during the reaction, leading to $\mathbf{2 a - f}$ in $54-83 \%$ yield. The presence of methyl acrylate or pyridine was also well-tolerated, as exemplified in the formation of $\mathbf{2 g}, \mathbf{h}$ in $48-57 \%$ yield. Besides methyl propargyl ethers, propargyl acetates were also tolerated under these conditions $(\mathbf{2} \mathbf{i}-\mathbf{k})$. The presence of substituents on the aromatic ring, such as a methyl group or a chlorine atom, did not have much of an effect the reaction $(\mathbf{2} \mathbf{j}, \mathbf{k})$.<smiles></smiles>

DBU $50^{\circ} \mathrm{C}, \mathrm{THF}, 12 \mathrm{~h}$<smiles>C=CCC(c1ccc(C(C)=O)cc1)c1sc2ccccc2c1OC</smiles>

2b $79 \%$<smiles>C=CCC(c1ccc(C(F)(F)F)cc1)c1sc2ccccc2c1OC</smiles>

2e $54 \%$<smiles>C=CCC(c1ccncc1)c1sc2ccccc2c1OC</smiles>

2h $57 \%$<smiles>C=CCC(c1ccc(Cl)c(Cl)c1)c1sc2ccccc2c1OC</smiles>

2c $71 \%$<smiles>C=CCC(c1ccc(C#N)cc1)c1sc2ccccc2c1OC</smiles>

2f $64 \%$<smiles>C=CC1C(=O)Oc2c(sc3ccccc23)C1c1ccc(C(=O)OCCCCCCCCCC)cc1</smiles>

$\operatorname{2g} 48 \%$<smiles>C=CC(=O)OC</smiles><smiles>C=CC1C(=O)Oc2c(sc3cc(C)ccc23)C1c1ccc(C(=O)OC)cc1</smiles>

2j $78 \%$<smiles>C=CC1C(=O)Oc2c(sc3cc(Cl)ccc23)C1c1ccc(C(=O)OC)cc1</smiles>

2k $67 \%$ 
Indoles are also important heterocycles that are the key motif of many natural products and pharmaceuticals. Consequently, new and straightforward methods to access indoles are highly desirable $[35,36]$. We chose a propargyl phosphite rearrangement to achieve allenyl intermediates and aimed to synthetize indoles via allenyl phosphonates, which were versatile synthetic intermediates [37,38]. The $N$-methyl- $N$-allylpropargyl alcohol 3 was treated with $(\mathrm{EtO})_{2} \mathrm{PCl}$ under alkaline conditions, then underwent a propargyl phosphite/allenyl phosphonate rearrangement and an intramolecular nucleophilic attack to form the indole moiety, followed by allyl migration (Scheme 2). Phosphinesubstituted indole derivatives were obtained in moderate yield (Figure 3, 4a-c).

\section{Conclusion}

In summary, we have developed an expedient route for the construction of benzothiophene and indole derivatives via propargyl-allene rearrangement and allyl migration. The reac- tion proceeded under mild conditions to produce useful benzothiophene and indole derivatives.

\section{Supporting Information}

\section{Supporting Information File 1}

Experimental procedures and analytical data.

[http://www.beilstein-journals.org/bjoc/content/ supplementary/1860-5397-13-181-S1.pdf]

\section{Acknowledgements}

We gratefully acknowledge the National Natural Science Foundation of China (Project No. 21606080), Zhejiang Provincial Natural Science Foundation of China (No. LY14B020008), the Ph.D. Scientific Research Starting Foundation of Jiaxing University (No: 70515007) and Summit Program of Jiaxing University for Leading Talents.<smiles>C=CCN(C)c1ccccc1C(O)C#Cc1ccccc1</smiles>

3

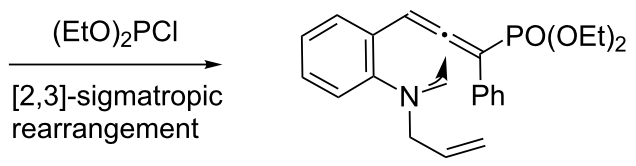

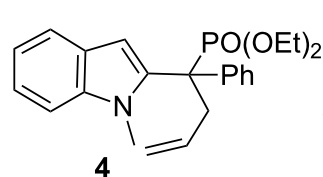

4<smiles>C=CCN(C)c1cc[R](F)cc1C(O)C#CCN(C)CC</smiles><smiles>[R][R]OCC(CC=C)(c1cc2ccccc2n1C)C(OCC)(c1ccccc1)c1ccccc1</smiles><smiles>C=CCC(c1cc2ccccc2n1C)(P(=O)(O)c1ccccc1)P(=O)(OCC)c1ccccc1</smiles>

4a $68 \%$<smiles>C=CCC(c1cc2ccc(C)cc2n1C)(p1oc2ccccc21)P(=O)(OCC)c1ccccc1</smiles>

4b $62 \%$<smiles>C=CCC(c1cc2cc(Br)ccc2n1C)(P(=O)(OCC)c1ccccc1)P(=O)(OCC)c1ccccc1</smiles>

4c $51 \%$

Figure 3: Synthesis of 1-methylindole phosphine oxides. Reaction conditions: $3(0.5 \mathrm{mmol}),(\mathrm{EtO})_{2} \mathrm{PCl}(0.6 \mathrm{mmol}), \mathrm{Et} \mathrm{t}_{3} \mathrm{~N}(1.5 \mathrm{mmol})$, and $\mathrm{THF}$ $(2.0 \mathrm{~mL})$ at $-78^{\circ} \mathrm{C}$. Yields are isolated yield. 


\section{References}

1. Deiters, A.; Martin, S. F. Chem. Rev. 2004, 104, 2199-2238. doi:10.1021/cr0200872

2. Zeni, G.; Larock, R. C. Chem. Rev. 2006, 106, 4644-4680. doi:10.1021/cr0683966

3. Curran, D. P.; Solovyev, A.; Brahmi, M. M.; Fensterbank, L.; Malacria, M.; Lacôte, E. Angew. Chem., Int. Ed. 2011, 50, 10294-10317. doi:10.1002/anie.201102717

4. Yamaguchi, J.; Yamaguchi, A. D.; Itami, K. Angew. Chem., Int. Ed. 2012, 51, 8960-9009. doi:10.1002/anie.201201666

5. Wu, X.-F.; Neumann, H.; Beller, M. Chem. Rev. 2012, 113, 1-35. doi:10.1021/cr300100s

6. Dubrovskiy, A. V.; Markina, N. A.; Larock, R. C. Org. Biomol. Chem. 2013, 11, 191-218. doi:10.1039/C2OB26673C

7. Joshi, E. M.; Heasley, B. H.; Chordia, M. D.; Macdonald, T. L. Chem. Res. Toxicol. 2004, 17, 137-143. doi:10.1021/tx0341409

8. Li, H.; Xiao, H.; Lin, L.; Jou, D.; Kumari, V.; Lin, J.; Li, C. J. Med. Chem. 2014, 57, 632-641. doi:10.1021/jm401144z

9. Xiong, R.; Patel, H. K.; Gutgesell, L. M.; Zhao, J.; Delgado-Rivera, L.; Pham, T. N. D.; Zhao, H.; Carlson, K.; Martin, T.; Katzenellenbogen, J. A.; Moore, T. W.; Tonetti, D. A.; Thatcher, G. R. J. J. Med. Chem. 2016, 59, 219-237. doi:10.1021/acs.jmedchem.5b01276

10. Chen, C.-C.; Chen, C.-M.; Wu, M.-J. J. Org. Chem. 2014, 79, 4704-4711. doi:10.1021/j0500377v

11. Dhage, Y. D.; Shirai, T.; Arima, M.; Nakazima, A.; Hikawa, H.; Taichi Kusakabe, I. A.; Takahashi, K.; Kato, K. RSC Adv. 2015, 5, 42623-42627. doi:10.1039/C5RA05263G

12. Gabriele, B.; Mancuso, R.; Lupinacci, E.; Veltri, L.; Salerno, G.; Carfagna, C. J. Org. Chem. 2011, 76, 8277-8286. doi:10.1021/jo201471k

13. Aurelio, L.; Volpe, R.; Halim, R.; Scammells, P. J.; Flynn, B. L. Adv. Synth. Catal. 2014, 356, 1974-1978. doi:10.1002/adsc. 201400072

14. Duan, Z.; Ranjit, S.; Liu, X. Org. Lett. 2010, 12, 2430-2433. doi:10.1021/ol100816g

15. Matsumura, M.; Muranaka, A.; Kurihara, R.; Kanai, M.; Yoshida, K.; Kakusawa, N.; Hashizume, D.; Uchiyama, M.; Yasuike, S. Tetrahedron 2016, 72, 8085-8090. doi:10.1016/j.tet.2016.10.048

16. Warner, A. J.; Churn, A.; McGough, J. S.; Ingleson, M. J. Angew. Chem., Int. Ed. 2017, 56, 354-358. doi:10.1002/anie.201610014

17. Tejedor, D.; Méndez-Abt, G.; Cotos, L.; Garcia-Tellado, F. Chem. Soc. Rev. 2013, 42, 458-471. doi:10.1039/C2CS35311C

18. Braverman, S.; Cherkinsky, M. Top. Curr. Chem. 2007, 275, 67-101. doi:10.1007/128_047

19. Chen, D.; Xing, G.; Yao, J.; Zhou, H. Org. Chem. Front. 2017, 4, 1042-1045. doi:10.1039/C6Q000675B

20. Chen, D.; Xing, G.; Yao, J.; Zhou, H. RSC Adv. 2016, 6, 103320-103323. doi:10.1039/C6RA21889J

21. Chen, D.; Xing, G.; Zhou, H. Org. Chem. Front. 2015, 2, 947-950. doi: $10.1039 / C 5 Q 000089 \mathrm{~K}$

22. Chen, D.; Xing, G.; Chen, X.; Yao, J.; Zhou, H. Tetrahedron Lett. 2016, 57, 5124-5126. doi:10.1016/j.tetlet.2016.10.025

23. Liu, L.; Wang, J.; Zhou, H. J. Org. Chem. 2015, 80, 4749-4753. doi:10.1021/acs.joc.5b00261

24. Liu, L.; Chen, D.; Zhou, H. Adv. Synth. Catal. 2015, 357, 389-394. doi:10.1002/adsc.201400713

25. Zhao, G.; Zhang, Q.; Zhou, H. J. Org. Chem. 2014, 79, 10867-10872. doi:10.1021/jo501867h
26. Zhao, G.; Zhang, Q.; Zhou, H. Adv. Synth. Catal. 2013, 355 , 3492-3496. doi:10.1002/adsc.201300573

27. Zhou, H.; Liu, L.; Xu, S. J. Org. Chem. 2012, 77, 9418-9421. doi:10.1021/jo3018186

28. Wang, C.; Kong, L.; Li, Y.; Li, Y. Eur. J. Org. Chem. 2014, 3556-3560. doi:10.1002/ejoc.201402059

29. Thummanapelli, S. K.; Hosseyni, S.; Su, Y.; Akhmedov, N. G.; Shi, X Chem. Commun. 2016, 52, 7687-7690. doi:10.1039/C6CC03032G

30. You, X.; Xie, X.; Chen, H.; Li, Y.; Liu, Y. Chem. - Eur. J. 2015, 21, 18699-18705. doi:10.1002/chem.201503374

31. Wang, T.; Naredla, R. R.; Thompson, S. K.; Hoye, T. R. Nature 2016, 532, 484-488. doi:10.1038/nature17429

32. Gobé, V.; Dousset, M.; Retailleau, P.; Gandon, V.; Guinchard, X. Adv. Synth. Catal. 2016, 358, 3960-3965. doi:10.1002/adsc. 201601075

33. Li, D.-Y.; Wei, Y.; Marek, I.; Tang, X.-Y.; Shi, M. Chem. Sci. 2015, 6, 5519-5525. doi:10.1039/C5SC01806D

34. Ji, K.-G.; Shu, X.-Z.; Chen, J.; Zhao, S.-C.; Zheng, Z.-J.; Lu, L.; Liu, X.-Y.; Liang, Y.-M. Org. Lett. 2008, 10, 3919-3922. doi:10.1021/ol8015463

35. Jin, H.; Huang, L.; Xie, J.; Rudolph, M.; Rominger, F.; Hashmi, A. S. K. Angew. Chem., Int. Ed. 2016, 55, 794-797. doi:10.1002/anie.201508309

36. Yan, H.; Wang, H.; Li, X.; Xin, X.; Wang, C.; Wan, B. Angew. Chem., Int. Ed. 2015, 54, 10613-10617. doi:10.1002/anie.201503997

37. Shen, R.; Luo, B.; Yang, J.; Zhang, L.; Han, L.-B. Chem. Commun. 2016, 52, 6451-6454. doi:10.1039/C6CC02563C

38. Hu, G.; Shan, C.; Chen, W.; Xu, P.; Gao, Y.; Zhao, Y. Org. Lett. 2016, 18, 6066-6069. doi:10.1021/acs.orglett.6b03028

\section{License and Terms}

This is an Open Access article under the terms of the Creative Commons Attribution License (http://creativecommons.org/licenses/by/4.0), which permits unrestricted use, distribution, and reproduction in any medium, provided the original work is properly cited.

The license is subject to the Beilstein Journal of Organic Chemistry terms and conditions:

(http://www.beilstein-journals.org/bjoc)

The definitive version of this article is the electronic one which can be found at: $\underline{\text { doi:10.3762/bjoc. } 13.181}$ 